\title{
The role of nicotinamide in acne treatment
}

\author{
Frances M. Walocko ${ }^{1}$ \\ Ariel E. Eber ${ }^{2}$ | Jonette E. Keri2,3 \\ Mana A. AL-Harbi ${ }^{4}$ \\ Keyvan Nouri ${ }^{2}$
}

${ }^{1}$ University of Michigan Medical School, Ann Arbor, Michigan

${ }^{2}$ Department of Dermatology \& Cutaneous Surgery, University of Miami Miller School of Medicine, Miami, Florida

${ }^{3}$ Miami VA Hospital, Miami, Florida

${ }^{4}$ Imam Muhammad ibn Saud I. University (IMSIU), Riyadh, Saudi Arabia

Correspondence

Frances M. Walocko, MSE, University of Michigan Medical School, M4101 Medical

Science Building I - C Wing, 1301

Catherine, Ann Arbor, MI 48109-5624.

Email: fwalocko@med.umich.edu

\begin{abstract}
Safe and effective treatment options for acne vulgaris are needed to address side effects and increasing rates of antibiotic resistance from current treatments. Nicotinamide is a vitamin with potent anti-inflammatory properties that could offer a potential treatment option. We aim to summarize the relevant literature on the role of nicotinamide in acne vulgaris and discuss the next steps necessary to move this approach into clinical practice. We searched PubMed for clinical studies using nicotinamide for treatment of acne vulgaris. We summarized the 10 studies that met our search criteria. Six of eight studies using topical nicotinamide led to a significant reduction in acne compared with the patient's baseline or performed similarly to another standard-of-care acne treatment. Both studies using an oral supplement containing nicotinamide resulted in a significant reduction in acne compared with baseline. No major adverse side effects were noted. Our review suggests that topical and oral nicotinamide has an unclear effect on acne vulgaris due to the limited nature of the available literature. Additional studies are needed comparing nicotinamide to other first-line acne treatments and evaluating the efficacy and side effect profile of nicotinamide over an extended period of time.
\end{abstract}

\section{KEYWORDS}

acne vulgaris, antibiotic resistance, inflammatory acne, niacinamide, nicotinamide, vitamin B3

\section{1 | INTRODUCTION}

Acne vulgaris is an extremely common chronic inflammatory disease of the skin that occurs in approximately $80 \%$ of young adults and adolescents, though it can occur in all ages (Fox, Csongradi, Aucamp, du Plessis, \& Gerber, 2016). While the pathogenesis of acne vulgaris is complex, the basic physiology is widely accepted. Hypercornification combined with excess sebum production provokes clogging of the pores (Toyoda \& Morohashi, 2001). Propionobacterium acnes ( $P$. acnes) proliferate in this environment and cause an increase in inflammatory cytokines and free fatty acids, which then leads to further irritation (Muizzuddin, Giacomoni, \& Maes, 2008). Studies suggest genetic, neuroendocrine, and dietary factors

Abbreviations: g, grams; mg, milligrams; mcg, micrograms; P. acnes, Propionibacterium acnes; NAD, nicotinamide adenine dinucleotide; NADP, nicotinamide adenine dinucleotide phosphate; PARP-1, poly-ADP-ribose polymerase-1. may also contribute to the multifactorial process of acne vulgaris pathogenesis (Zaenglein et al., 2016).

Treatments for acne vulgaris target one or more of the steps in pathogenesis and may be administered topically or orally. The most widely used acne treatments are topical formulations. Common examples include retinoids, antibiotics, antibacterial agents, and comedolytic agents (Fox et al., 2016). Systemic treatments are used for more severe or resistant forms of acne, including oral isotretinoin, oral antibiotics, and hormonal agents (Katsambas \& Papakonstantinou, 2004). These treatment modalities are not without their drawbacks. Topical retinoid use may be limited by dryness, erythema, and irritation, while oral isotretinoin treatment is teratogenic and requires routine monitoring of serum lipid profiles and liver function tests (McElwee et al., 1991). Topical and oral antibiotic overuse and resistance are a growing global concern, both for the effectiveness of acne therapies and for the treatment of infectious diseases (Humphrey, 2012; Ventola, 2015). Many countries have reported that over $50 \%$ of $P$. acnes strains are antibiotic resistant, particularly to topical macrolides (Walsh, Efthimiou, 
\& Dreno, 2016). Regarding the use of hormonal modulators, venous thromboembolism risk remains a concern. For these reasons, alternative treatments have entered the arena of acne care.

Nicotinamide provides potent anti-inflammatory properties without the risk of bacterial resistance and systemic side effects and represents a potential treatment modality for acne vulgaris. Nicotinamide, also known as niacinamide, is a form of vitamin B3, which is an essential water-soluble nutrient present in a variety of foods. Nicotinamide is the amide form of nicotinic acid (niacin) and is identical in vitamin function; however, it does not carry the same vasodilatory, hypolipidemic, and gastrointestinal actions (Knip et al., 2000). Furthermore, nicotinamide has demonstrated a low incidence of side effects and toxicity at oral doses up to $3 \mathrm{~g}$ per day for up to 5 years (Knip et al., 2000). Nicotinamide is a precursor for nicotinamide adenine dinucleotide (NAD) and the phosphorylated derivative NADP (Hoffer, 1967). NAD provides a substrate for nuclear enzyme poly-ADP-ribose polymerase (PARP-1), which repairs damage from genotoxic stresses, such as UV radiation (Park, Halliday, Surjana, \& Damian, 2010; Surjana, Halliday, \& Damian, 2010). Having adequate cellular energy and properly functioning PARP-1 is important for a number of skin conditions, for which nicotinamide may have beneficial effects (Chen et al., 2015).

Nicotinamide appears to play a number of potential roles in the treatment of acne vulgaris. Draelos et al. demonstrated that $2 \%$ topical nicotinamide resulted in a significant reduction in sebum excretion rate in a Japanese study group and decreased casual sebum levels (sebum on the skin surface) in a Caucasian study group over 4 weeks (Draelos, Matsubara, \& Smiles, 2006). In addition, topical nicotinamide helps protect the natural barrier of the skin against infection and may have a bacteriostatic effect on P. acnes (Fivenson, 2006; Wohlrab \& Kreft, 2014). Finally, nicotinamide decreases the in vitro secretion of interleukin-8, a cytokine secreted by keratinocytes in response to pathogens (including $P$. acnes), thereby exerting an anti-inflammatory effect through inhibition of leukocyte chemotaxis (Grange, Raingeaud, Calvez, \& Dupin, 2009). Additional anti-inflammatory action may occur via inhibition of lysosomal enzyme release and mast cell degranulation (Wohlrab \& Kreft, 2014).

Nicotinamide for use in dermatologic ailments has shown promise in clinical studies but has not yet become a standard treatment option. This review examines the potential role of nicotinamide in acne vulgaris, summarizes the relevant published articles, and discusses what studies are needed to move this new approach to such a common ailment into widespread clinical practice.

\section{2 | METHODS}

We searched PubMed for all articles in English with the following words in the title or abstract: "acne," "nicotinamide," "niacinamide," and "vitamin B3" with no restriction on dates up to October 2016. This search yielded 41 articles. Two reviewers independently determined the eligibility of the studies and performed the methodological quality assessment.
Inclusion Criteria:

- Studies must directly involve subjects with at least mild acne

- Studies must use a human model

- Studies must use topical and/or oral nicotinamide treatments

For each experiment, we determined the degree of acne vulgaris, treatment group regimen, control group regimen, duration of treatment, outcome measures, and results. Treatments containing only nicotinamide are referred to as "single-agent products," whereas treatments containing nicotinamide in addition to other components are referred to as "combination products." Quantitative assessment of acne lesions involved counts of comedones, papules/pustules, and/or cysts. Qualitative assessment of acne lesions was based on the Physician's Global Evaluation of Inflammatory Acne or modifications of Cook's Acne Grade. The Physician's Global Evaluation of Inflammatory Acne involves rating the change in acne on a scale from "much better" to "worse." Cook's Acne Grade involves rating acne based on how much of the face is involved. We summarized our findings and determined what essential information is missing from the literature, and thus, should be studied before nicotinamide can make its way into standard clinical treatment of acne vulgaris.

\section{3 | RESULTS}

Ten articles matched our search criteria; all studies tested the efficacy of nicotinamide on acne vulgaris in humans using either topical or oral therapies. The design and results of the studies are summarized in Tables 1-3. Eight out of the 10 studies demonstrated a significant reduction in acne lesions compared with the patient's baseline acne or similar efficacy to a commonly used treatment for acne vulgaris. Four of the studies used nicotinamide as a single-agent topical product; four of the studies used nicotinamide as a combination topical product; and two of the studies used an oral form of nicotinamide. Outcomes of the available research for each study design are summarized below.

\section{1 | Single-agent topical products}

Four studies used nicotinamide as a single-agent topical product (Table 1). Three of the studies randomized patients to treatment with nicotinamide only or clindamycin only; all three studies found that the nicotinamide group had significantly improved acne vulgaris from baseline and that nicotinamide and clindamycin resulted in similar reductions in acne lesions (Khodaeiani, Fouladi, Amirnia, Saeidi, \& Karimi, 2013; Shahmoradi, Iraji, Siadat, \& Ghorbaini, 2013; Shalita, Smith, Parish, Sofman, \& Chalker, 1995). Kaymak treated all study participants with only niacinamide and found a significant improvement in acne vulgaris from baseline (Kaymak, 2008). None of the four studies compared nicotinamide to a placebo-treated control group.

\subsection{Combination topical products}

Four studies used nicotinamide in a combination topical product (Table 2). Dos et al. and Sardesai and Kambli directly compared a 

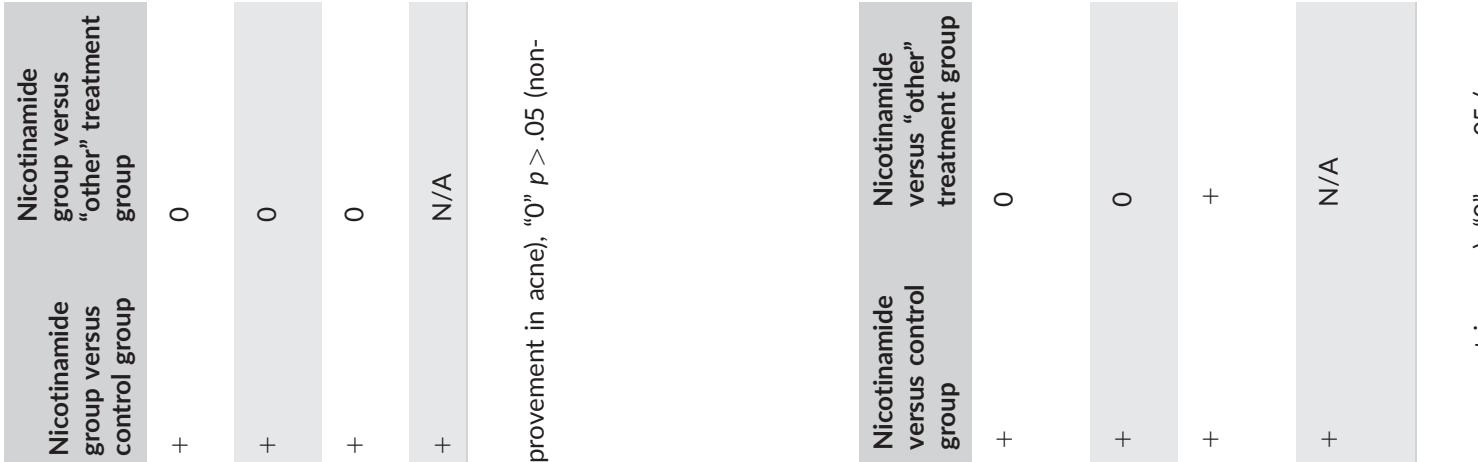

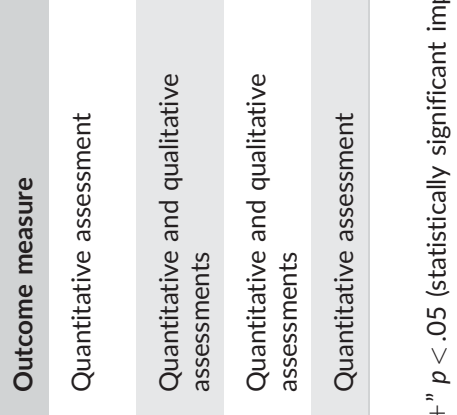

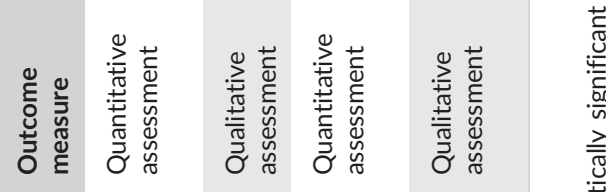

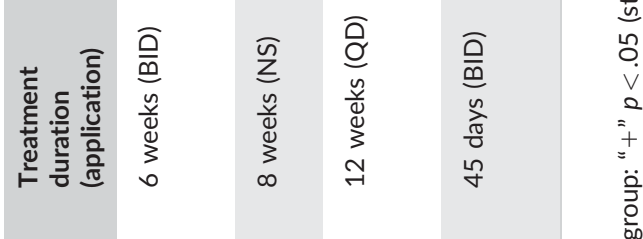
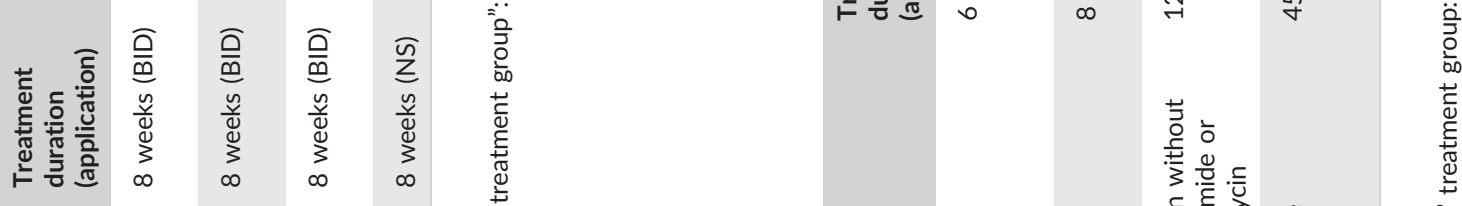

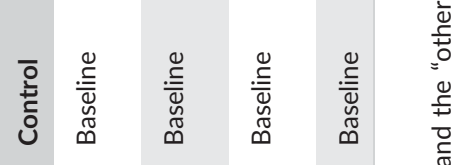
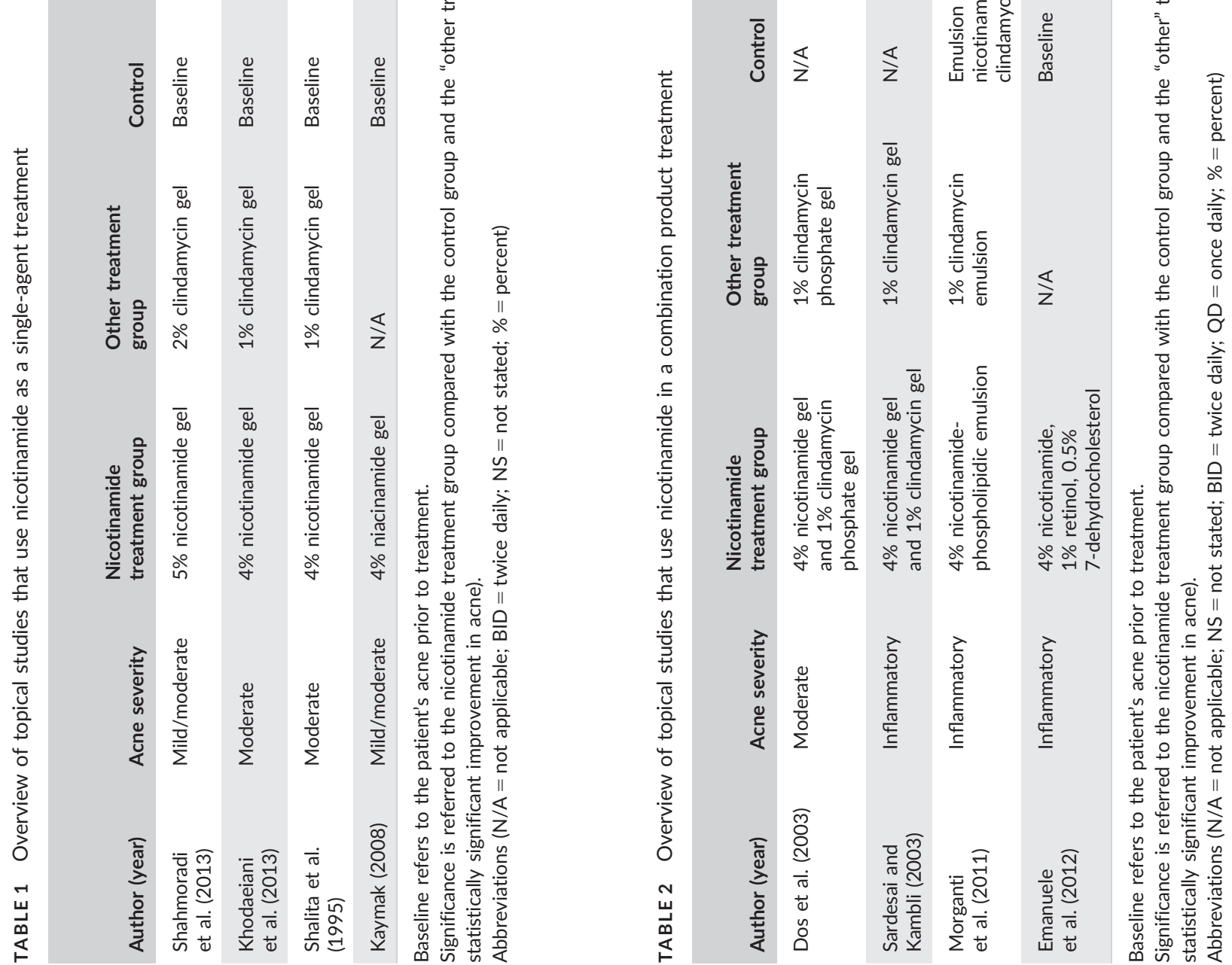


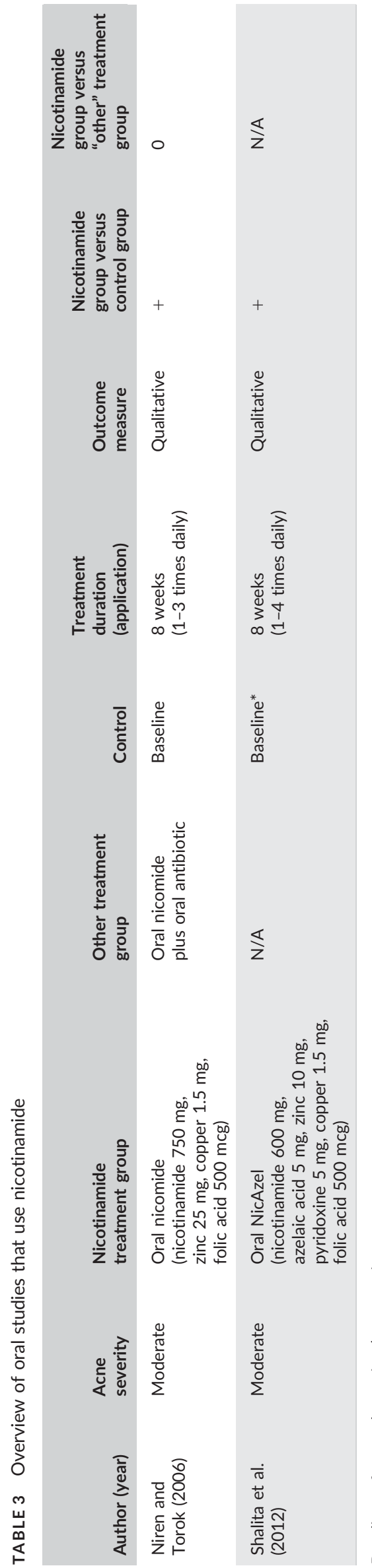

combination product containing nicotinamide and clindamycin to a clindamycin-only treatment group (Dos, Barbhuiya, Jana, \& Dey, 2003; Sardesai \& Kambli, 2003). Both studies demonstrated a significant improvement in acne vulgaris from baseline for both treatment groups, but there was no difference in acne vulgaris when topical nicotinamide and clindamycin were combined (Dos et al., 2003; Sardesai \& Kambli, 2003). Morganti and colleagues randomized patients to receive treatment with emulsions composed of numerous ingredients and either $4 \%$ nicotinamide, $1 \%$ clindamycin, or neither (emulsion vehicle) (Morganti et al., 2011). The nicotinamide treatment group quantitatively demonstrated a significant improvement in acne vulgaris compared with the emulsion containing clindamycin and the emulsion vehicle (Morganti et al., 2011). Emanuele compared a nicotinamide treatment group containing $4 \%$ nicotinamide, $1 \%$ retinol, and $0.5 \%$ 7-dehydrocholesterol to the patient's baseline acne (Emanuele, Bertona, Altabas, Altabas, \& Alessandrini, 2012). There was a significant improvement in acne vulgaris using this treatment compared with the patient's baseline (Emanuele et al., 2012). It is unclear if the nicotinamide or the other ingredients in the combination product were responsible for the significant improvement.

\section{3 | Oral nicotinamide products}

Two studies involving oral nicotinamide therapy for acne vulgaris matched our search criteria (Table 3). Niren and Torok used a combination product containing nicotinamide, zinc, copper, and folic acid and demonstrated a significant improvement in acne vulgaris compared with acne prior to treatment (Niren \& Torok, 2006). There was no difference in acne lesions when an oral antibiotic (name not stated in primary article) was combined with the nicotinamide combination product. Shalita et al. used a combination product containing nicotinamide, azelaic acid, zinc, pyridoxine, copper, and folic acid and showed a significant improvement in acne vulgaris compared with acne prior to treatment (Shalita et al., 2012). Participants in this study were permitted to continue their regular acne regimens during the study; therefore, it is unclear if the treatment was responsible for the improvement in acne vulgaris. In both studies, the individual components of each combination product were not tested separately; therefore, it is unclear if nicotinamide itself contributed to the improvement in acne vulgaris. There were no studies that tested the effect of oral nicotinamide on acne vulgaris as a single-agent.

\subsection{Side effects}

No major adverse side effects were noted by any of the studies. Minor side effects for nicotinamide topical treatments included itching, burning, mild dermatitis, and greasy skin. These side effects were also experienced by patients in the "other topical treatment" groups with no significant difference in frequency or severity between groups in any of the topical studies. No side effects were experienced by patients taking oral nicotinamide formulations. 


\section{DISCUSSION}

Acne vulgaris remains a prevalent problem, and the need persists for new safe and effective treatment options. Current modalities carry significant risks, including antibiotic resistance and systemic interactions (Walsh et al., 2016). Unpleasant side effects, such as dry, irritated skin with topical retinoids or mandatory routine laboratory monitoring with oral isotretinoin, are not only concerning, but they limit compliance for many patients. Topical and oral forms of nicotinamide represent a possible treatment alternative for acne vulgaris, but the benefit of these products is unclear. Our literature review suggests that a limited series of studies demonstrate that topical nicotinamide treatments have a significant effect on reducing acne vulgaris with minimal side effects. Four of these studies also showed that topical nicotinamide reduced acne lesions similarly to topical clindamycin, thereby implying that nicotinamide could potentially be used as a treatment alternative to clindamycin. However, two of the eight topical studies showed that nicotinamide did not demonstrate a reduction in acne lesions when added to clindamycin treatment. The benefit of oral nicotinamide is further unclear because nicotinamide has only been tested as a combination product and not by itself.

The authors opine that there are several study design limitations that need to be addressed to determine if nicotinamide is a potential treatment option for acne vulgaris in the clinical setting. The current literature would benefit from additional studies using an adequate control group, expanding comparisons of nicotinamide with other standard-of-care acne vulgaris treatments, lengthening treatment endpoints, objectifying outcome measures, and establishing a long-term side effect profile.

\section{1 | Control groups}

Six of the 10 studies examined combination treatments without including a treatment group consisting of only nicotinamide. This design makes it difficult to determine what product within the combination treatment is responsible for the effect on acne vulgaris. In addition, only one study prospectively randomized patients to a control group that received treatment with a gel containing the active treatment or a vehicle gel (gel that does not contain active treatment); including such a control group would clarify if the improvement was due to time or the treatment itself (Morganti et al., 2011).

\section{2 | Additional treatment groups}

The American Academy of Dermatology's treatment algorithm for acne vulgaris recommends first line treatment for mild acne with benzoyl peroxide, topical retinoid, or topical combination therapy (benzoyl peroxide + antibiotic, retinoid + benzoyl peroxide, or retinoid + benzoyl peroxide + antibiotic) (Zaenglein et al., 2016). These agents are often used in the treatment of moderate acne vulgaris as well. Therefore, there is a need to compare the effects of nicotinamide to these first-line products in single treatment and combination treatment forms. No topical studies compared nicotinamide to benzoyl peroxide or used a nicotinamide-benzoyl peroxide combination product. One topical nicotinamide combination product included retinol but did not directly compare nicotinamide by itself to a retinoid gel or a vehicle gel. None of the oral nicotinamide studies compared nicotinamide to systemic antibiotics alone or to oral isotretinoin.

The optimal concentrations, doses, and treatment regimens have yet to be determined for topical and oral nicotinamide treatments. Topical nicotinamide gel has shown positive effects on acne vulgaris at $4 \%$ and $5 \%$ concentrations. Oral nicotinamide has been used successfully to treat acne vulgaris at $600 \mathrm{mg}$ and $750 \mathrm{mg}$ nicotinamide one to four times daily (Niren \& Torok, 2006). Further studies are needed to directly compare different amounts of nicotinamide to obtain a clinical standard for acne vulgaris treatment.

\subsection{Study length}

Only one topical study tested treatment groups for to an endpoint of 12 weeks (Morganti et al., 2011). The other topical studies all used endpoints ranging from 6 to 8 weeks. Clindamycin was used as a comparison group in six out of the eight topical studies; however, the topical form of this antibiotic is effective at reducing acne lesions over an 8- to 12-week period (Shahlita, Smith, \& Bauer, 1984). Combination products consisting of clindamycin and benzoyl peroxide have shown significant treatment benefit over an 11-week period, while topical retinoids have shown benefit over a 12-week period (Berger et al., 2007; Jarratt \& Brundage, 2012; Lookingbill et al., 1997). Additional studies with endpoints of at least 12 weeks for nicotinamide treatment are needed to determine the true efficacy of topical nicotinamide treatment compared with these agents.

Both oral nicotinamide treatments were tested for 8 weeks and compared with baseline (Niren \& Torok, 2006; Shalita et al., 2012). However, most systemic antibiotics and oral isotretinoin treatments are given over a course of 3-6 months (Akman et al., 2007; Kus, Yucelten, \& Aytug, 2005). Future studies involving oral nicotinamide treatments should consider expanding their treatment course to further establish long-term effects on acne vulgaris treatment. Additional studies are needed for both oral and topical treatments that detail efficacy following cessation of treatment.

\section{4 | Outcome measures}

Eight of the 10 studies used quantitative outcome measures, but there was little consistency in the outcome measures used (number of lesions, scarring, etc.). Although no universal grading scale can be recommended, using a quantitative grading scale that includes the numbers and type of acne lesions, disease severity, and scarring can help objectively guide treatment over time and compare results between treatment options (Zaenglein et al., 2016). Improvement in erythema and reduction in sebum production are additional outcome measures that could help define responses to nicotinamide treatment (Winston \& Shalita, 1991). 


\subsection{Side effect profile}

In this review, no major adverse effects were noted by patients receiving treatment with nicotinamide; however, longer term studies are needed to adequately profile side effects of nicotinamide for both topical and oral agents. Although most patients experience minimal side effects at topical concentrations less than $6 \%$ and oral doses less than $3 \mathrm{~g} /$ day, potential severe side effects at high doses include elevation of liver enzymes, nausea, heartburn, and flushing (Knip et al., 2000).

\section{5 | FUTURE APPLICATIONS}

Nicotinamide has shown promise not only in the realm of treating acute acne vulgaris, but also with respect to complications of acne, including pigmentation changes. Recent studies have demonstrated that nicotinamide has significant skin lightening effects through the inhibition of melanosome transfer from melanocytes to keratinocytes (Hakozaki et al., 2002). This mechanism of action could benefit patients being treated for acne who are predisposed to post-inflammatory hyperpigmentation, but future studies are needed. Furthermore, nicotinamide may not cause sun-sensitivity that is associated with other lightening agents used to treat post-inflammatory hyperpigmentation. Rather, nicotinamide potentially prevents photoimmunosuppression and photocarcinogenesis (Chen et al., 2015). These benefits make it a possible adjunct therapy to existing photosensitizing acne treatments, such as the tetracycline antibiotics or retinoids. Uses of nicotinamide should continue to be explored through randomized controlled clinical trials as it has the potential to benefit dermatologic care for multiple indications.

\section{6 | LIMITATIONS OF THIS REVIEW}

Our study has several limitations. First, as this is a review article, there is a potential for publication bias. If only positive studies are published, the review of the literature will be overwhelmingly positive. This is an inherent problem for all review articles and could very well be a factor in this review. However, there has been increasing awareness of this issue, and many studies with negative results are being published in high impact journals. Second, our review did not limit our included studies to only double-blinded randomized-controlled trials due to the lack of such studies in the literature. Therefore, the results reported in the studies are more subject to biases and less clearly due to the effects of nicotinamide. However, the findings by multiple studies that nicotinamide had an equivalent effect as clindamycin certainly supports more thorough investigation into the treatment potential of nicotinamide. Finally, because only 10 studies met our selection criteria and most of the studies were relatively small, we may simply not have enough power to demonstrate real differences between nicotinamide and clindamycin. This further highlights the need for large, double-blinded, randomized-controlled clinical trials.

\section{7 | CONCLUSION}

Nicotinamide is an inexpensive, over-the-counter, water-soluble vitamin with a well-established safety profile. Limited studies have sug- gested that it is effective in treating acne vulgaris, but additional evidence is necessary before nicotinamide can be considered an alternative or adjunct to current acne vulgaris treatment regimens. Additional randomized controlled clinical trials are needed comparing nicotinamide to other first-line acne treatments and evaluating the efficacy and the side effect profile of nicotinamide over an extended period of time.

\section{ACKNOWLEDGMENT}

None.

\section{CONFLICTS OF INTEREST}

All of the authors have no financial support or conflicts of interest to disclose for this manuscript.

\section{ORCID}

Frances M. Walocko (D) http://orcid.org/0000-0001-5723-6258

\section{REFERENCES}

Akman, A., Durusoy, C., Senturk, M., Koc, C. K., Soyturk, D., \& Alpsoy, E. (2007). Treatment of acne with intermittent and conventional isotretinoin: A randomized, controlled multicenter study. Archives of Dermatological Research, 299, 467-473.

Berger, R., Barba, A., Fleischer, A., Leyden, J. J., Lucky, A., Pariser, D., ..., \& Nighland, M. (2007). A double-blinded, randomized, vehiclecontrolled, multicenter, parallel-group study to assess the safety and efficacy of tretinoin gel microsphere $0.04 \%$ in the treatment of acne vulgaris in adults. Cutis, 80, 152-157.

Chen, A. C., Martin, A. J., Choy, B., Fernández-Peñas, P., Dalziell, R. A., McKenzie, C. A., ..., \& St. George, G. (2015). A phase 3 randomized trial of nicotinamide for skin-cancer chemoprevention. The New England Journal of Medicine, 373, 1618-1626.

Dos, S. K., Barbhuiya, J. N., Jana, S., \& Dey, S. K. (2003). Comparative evaluation of clindamycin phosphate $1 \%$ and clindamycin phosphate $1 \%$ with nicotinamide gel $4 \%$ in the treatment of acne vulgaris. Indian Journal of Dermatology, Venereology and Leprology, 69, 8-9.

Draelos, Z. D., Matsubara, A., \& Smiles, K. (2006). The effect of $2 \%$ niacinamide on facial sebum production. Journal of Cosmetic and Laser Therapy: Official Publication of the European Society for Laser Dermatology, 8, 96-101.

Emanuele, E., Bertona, M., Altabas, K., Altabas, V., \& Alessandrini, G. (2012). Anti-inflammatory effects of a topical preparation containing nicotinamide, retinol, and 7-dehydrocholesterol in patients with acne: A gene expression study. Clinical, Cosmetic and Investigational Dermatology, 5, 33-37.

Fivenson, D. P. (2006). The mechanisms of action of nicotinamide and zinc in inflammatory skin disease. Cutis, 77, 5-10.

Fox, L., Csongradi, C., Aucamp, M., Du Plessis, J., \& Gerber, M. (2016). Treatment modalities for acne. Molecules, 21, 1063.

Grange, P. A., Raingeaud, J., Calvez, V., \& Dupin, N. (2009). Nicotinamide inhibits propionibacterium acnes-induced IL-8 production in keratinocytes through the NF-kappaB and MAPK pathways. Journal of Dermatological Science, 56, 106-112.

Hakozaki, T., Minwalla, L., Zhuang, J., Chhoa, M., Matsubara, A., Miyamoto, K., ..., \& Boissy, R. E. (2002). The effect of niacinamide 
on reducing cutaneous pigmentation and suppression of melanosome transfer. The British Journal of Dermatology, 147, 20-31.

Hoffer, A. (1967). Biochemistry of nicotinic acid and nicotinamide. Psychosomatics, 8, 95-100.

Humphrey, S. (2012). Antibiotic resistance in acne treatment. Skin Therapy Letter, 17, 1-3.

Jarratt, M. T., \& Brundage, T. (2012). Efficacy and safety of clindamycintretinoin gel versus clindamycin or tretinoin alone in acne vulgaris: A randomized, double-blind, vehicle-controlled study. Journal of Drugs in Dermatology, 11, 318-326.

Katsambas, A., \& Papakonstantinou, A. (2004). Acne: Systemic treatment. Clinics in Dermatology, 22, 412-418.

Kaymak, Y., \& Önder, M. (2008). An investigation of efficacy of topical Niacinamide for the treatment of Mild and moderate acne vulgaris. J Turk Acad Dermatol, 2(4).

Khodaeiani, E., Fouladi, R. F., Amirnia, M., Saeidi, M., \& Karimi, E. R. (2013). Topical $4 \%$ nicotinamide vs. $1 \%$ clindamycin in moderate inflammatory acne vulgaris. International Journal of Dermatology, 52, 999-1004.

Knip, M., Douek, I. F., Moore, W. P., Gillmor, H. A., McLean, A. E. M., Bingley, P. J., \& Gale, E. A. M. (2000). Safety of high-dose nicotinamide: A review. Diabetologia, 43, 1337-1345.

Kus, S., Yucelten, D., \& Aytug, A. (2005). Comparison of efficacy of azithromycin vs. doxycycline in the treatment of acne vulgaris. Clinical and Experimental Dermatology, 30, 215-220.

Lookingbill, D. P., Chalker, D. K., Lindholm, J. S., Katz, H. I., Kempers, S. E., Huerter, C. J., ..., \& Klauda, H. C. (1997). Treatment of acne with a combination clindamycin/benzoyl peroxide gel compared with clindamycin gel, benzoyl peroxide gel and vehicle gel: Combined results of two double-blind investigations. Journal of the American Academy of Dermatology, 37, 590-595.

McElwee, N. E., Schumacher, M., Johnson, S. C., Weir, T. W., Greene, S. L., Scotvold, M. J., ..., \& Jick, H. (1991). An observational study of isotretinoin recipients treated for acne in a health maintenance organization. Archives of Dermatology, 127, 341-346.

Morganti, P., Berardesca, E., Guarneri, B., Guarneri, F., Fabrizi, G., Palombo, P., \& Palombo, M. (2011). Topical clindamycin 1\% vs. linoleic acid-rich phosphatidylcholine and nicotinamide $4 \%$ in the treatment of acne: A multicentre-randomized trial. International Journal of Cosmetic Science, 33, 467-476.

Muizzuddin, N., Giacomoni, P., \& Maes, D. (2008). Acne-A multifaceted problem. Drug Discovery Today: Disease Mechanisms, 5, e183-e188.

Niren, N. M., \& Torok, H. M. (2006). The Nicomide Improvement in Clinical Outcomes Study (NICOS): Results of an 8-week trial. Cutis, 77, 17-28.

Park, J., Halliday, G. M., Surjana, D., \& Damian, D. L. (2010). Nicotinamide prevents ultraviolet radiation-induced cellular energy loss. Photochemistry and Photobiology, 86, 942-948.

Sardesai, V. R., \& Kambli, V. M. (2003). Comparison of efficacy of topical clindamycin and nicotinamide combination with plain clindamycin for the treatment of acne vulgaris and acne resistant to topical antibiotics. Indian Journal of Dermatology, Venereology and Leprology, 69, 138-139.

Shahlita, A. R., Smith, E. B., \& Bauer, E. (1984). Topical erythromycin v clindamycin therapy for acne. A multicenter, double-blind comparison. Archives of Dermatology, 120, 351-355.

Shahmoradi, Z., Iraji, F., Siadat, A. H., \& Ghorbaini, A. (2013). Comparison of topical $5 \%$ nicotinamid gel versus $2 \%$ clindamycin gel in the treatment of the mild-moderate acne vulgaris: A double-blinded randomized clinical trial. Journal of Research in Medical Sciences: The Official Journal of Isfahan University of Medical Sciences, 18, 115-117.

Shalita, A. R., Falcon, R., Olansky, A., lannotta, P., Akhavan, A., Day, D., ..., \& Kallal, J. E. (2012). Inflammatory acne management with a novel prescription dietary supplement. Journal of Drugs in Dermatology, 11, 1428-1433.

Shalita, A. R., Smith, J. G., Parish, L. C., Sofman, M. S., \& Chalker, D. K. (1995). Topical nicotinamide compared with clindamycin gel in the treatment of inflammatory acne vulgaris. International Journal of Dermatology, 34, 434-437.

Surjana, D., Halliday, G. M., \& Damian, D. L. (2010). Role of nicotinamide in DNA damage, mutagenesis, and DNA repair. Journal of Nucleic Acids, 2010, 157591

Toyoda, M., \& Morohashi, M. (2001). Pathogenesis of acne. Medical Electron Microscopy, 34, 29-40.

Ventola, C. L. (2015). The antibiotic resistance crisis: Part 1: Causes and threats. $P \&$ \&: A Peer-Reviewed Journal for Formulary Management, 40, 277-283.

Walsh, T. R., Efthimiou, J., \& Dreno, B. (2016). Systematic review of antibiotic resistance in acne: An increasing topical and oral threat. The Lancet Infectious Diseases, 16, e23-e33.

Winston, M. H., \& Shalita, A. R. (1991). Acne vulgaris. Pathogenesis and treatment. Pediatric Clinics of North America, 38, 889903.

Wohlrab, J., \& Kreft, D. (2014). Niacinamide-Mechanisms of action and its topical use in dermatology. Skin Pharmacology and Physiology, 27, 311-315.

Zaenglein, A. L., Pathy, A. L., Schlosser, B. J., Alikhan, A., Baldwin, H. E., Berson, D. S., ..., \& Keri, J. E. (2016). Guidelines of care for the management of acne vulgaris. Journal of the American Academy of Dermatology, 74, 945-973 e33.

How to cite this article: Walocko FM, Eber AE, Keri JE, ALHarbi MA, Nouri K. The role of nicotinamide in acne treatment. Dermatologic Therapy. 2017;30:e12481. https://doi.org/ 10.1111/dth.12481 